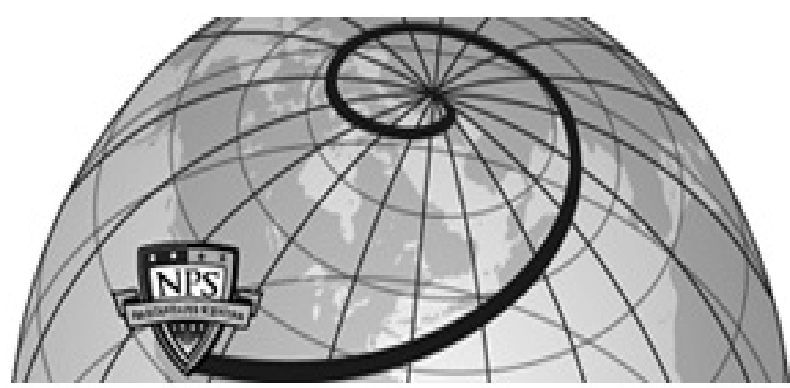

Calhoun: The NPS Institutional Archive DSpace Repository

\title{
A comparison between some methods for computing optimum paths in the problem of Bolza
}

Faulkner, Frank David.

Monterey, California. Naval Postgraduate School

https://hdl.handle.net/10945/31715

This publication is a work of the U.S. Government as defined in Title 17, United States Code, Section 101. Copyright protection is not available for this work in the United States.

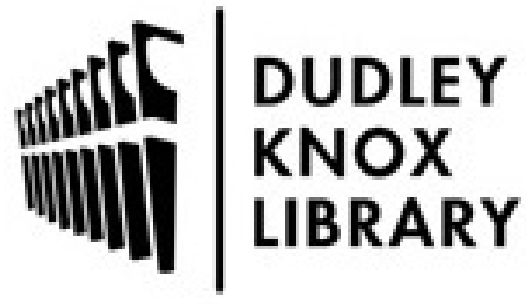

http://www.nps.edu/library
Calhoun is the Naval Postgraduate School's public access digital repository for research materials and institutional publications created by the NPS community. Calhoun is named for Professor of Mathematics Guy K. Calhoun, NPS's first appointed -- and published -- scholarly author.

Dudley Knox Library / Naval Postgraduate School 411 Dyer Road / 1 University Circle Monterey, California USA 93943 
Frank David Faulkner

A COMPARISON BETWEEN SOME METHODS FOR COMPUTING OPTIMUM

PATHS IN THE PROBLEM OF BOLZA. 


\section{Library}

U. S. Naval Postgraduate School

Monterey, California 


\section{UNITED STATES \\ NAVAL POSTGRADUATE SCHOOL MONTEREY, CALIFORNIA}

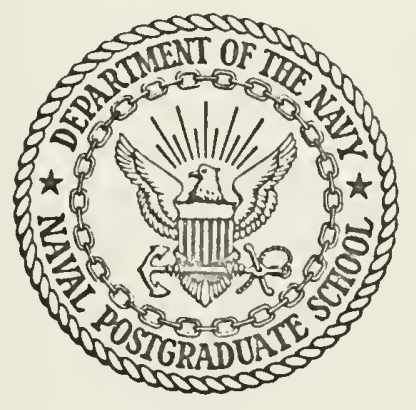

A COMPARISON BETWEEN SOME METHODS FOR COMPUTING OPTIMUM PATHS

IN THE PROBLEM OF BOLZA

By

Frank D. Faulkner

Research Paper No. 43

March 1964 

A COMPARISON BETWEEN SOME METHODS FOR COMPUTING OPTIMUM PATHS IN THE PROBLEM OF BOLZA

By

FRANK D. FAULKNER

Professor of Mathematics and Mechanics

UNITED STATES NAVAL POSTGRADUATE SCHOOL Monterey, California

Research Paper No. 43

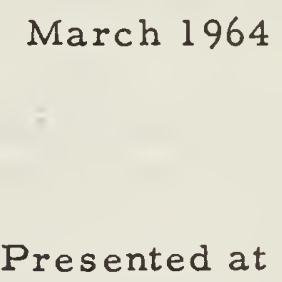

Conference in Computing Methods

in

Optimization Problems,

at

University of California

Los Angeles, California

January 1964 
TA7.

$u_{6}$

ho. 43 


\section{A COMPARISON BETWEEN SOME METHODS FOR COMPUTING OPTIMUM PATHS IN THE PROBLEM OF BOLZA}

Frank D. Faulkner, Department of Mathematics and Mechanics, U. S. Nava1 Postgraduate Schoo1, Monterey, California.

A comparison is made between methods for calculating optimum trajectories in the problem of Bolza. For the most part the comparison is between the method of steepest ascent as given by Bryson and Denham (1) and a direct method due to the author, using the differential techniques which G. A. Bliss developed during World War I in ballistics. A second purpose of the paper is to give a modification of Bryson and Denham's method which eliminates one of the minor subproblems and makes it possible to use a trajectory obtained by their method to start the routine for the other method. The reason for this will be given.

Both methods seem to yield reasonably satisfactory results and both have minor problems associated with actual computation, the most recurrent being that of convergence.

Both methods use the adjoint system of differential equations much as used by Bliss; the adjoint system seems imperative for the solution and understanding of all but the simplest problems.

Bryson and Denham's method, henceforth called the BFL method is based on the constructive proof of the fundamental lemma of the calculus of variations. This shows how, if an admissible path is given on which the Euler equations are not satisfied, a better admissible path can be obtained. A modification has been made up to eliminate the trouble of integrating forward and then backward. In the other method only extremals are used. Usually Bliss's differential methods are used to set up a Newton iteration for the constants, though they may also be obtained directly (see (2)). This method will be called the FD method.

The comparisons so far suggest the following conclusions. There are some advantages of the FD method. (1) It converges more rapidly, if it converges. It is a Newton iteration for the roots to a set of equations and tends to converge exponentially once the solution is approached. (2) There is no question of how closely the path approaches an extremal, since only extremals are used. This is often not too important from a practical point of view, since trajectories "near" the 


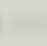

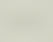

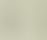
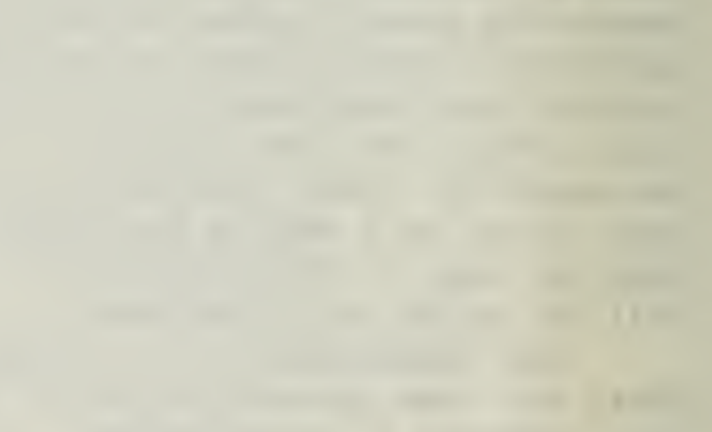
optimum are generally "almost" as good. (3) Discontinuous or even unbounded control and constraints on the variables seem to pose no major problem. (4) The optimum correction to a perturbed optimum trajectory involves only a few more iterations. (5) Various identities and relations associated with extremals can be used; for example, if the independent variable does not enter explicitly, the Hamiltonian is constant. (6) There is no arbitrary step size in the solution; they are determined by the Newton-Raphson routine. (7) The method seems more flexible in treating unusual problems. Solutions were obtained for the bang-bang control problem (3), for problems with discontinuous bounded control and further limitations on the energy in rocketry (4), (ㄷ), (6), and for problems with impulse solutions; simple allocation problems have also been treated by this method.

There are several advantages to the BFL method. (1) It seems to require less skill or luck in getting started. (2) The integral relations for the corrections are computationally more stable. They involve only first derivatives whereas the other method involves second derivatives with respect to the control variables. (3) It seems easier to get a convergent sequence of paths without interrupting the computation. Another FL method (that is, one based on the fundamental lemma) is due to Kelley (7); it was not investigated at length. It had the desirable feature of simplicity but it converged more slowly, perhaps due to inability of the author to select a sequence of parameters we 11 .

The methods also have their problems and difficulties. In the FD method the parameters are the constants of integration associated with the adjoint system of differential equations. The programmer may have no idea as to the proper range of these. The other method requires only a likely initial path; while even this may be hard to find, the uncertainty seems to be an order less. Usually the FD method must be modified if the strong Legendre condition is not satisfied. The methods based on the fundamental lemma are computationally more stable; in the direct method the control variable is obtained every step from equations which involve second derivatives in the denominator. In the FL methods both forward and backward integration are usually used. This, however, is only a matter of programing; a routine is given which eliminates this. Another problem associated with FL methods is that there is no obvious metric which indicates how much the path differs from an extremal. Finally, no way is seen to extend FL methods to problems involving discontinuous or bounded control generally.

Since FL methods are more likely to converge, but tend to converge more slowly and it is not clear when convergence Is-attained, it is desirable to get a "good" path using the 


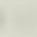

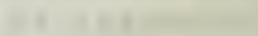

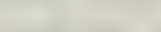

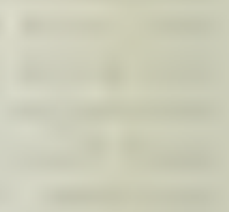

r.

. 
FL method, then use it to obtain a set of initial values for the Lagrange multipliers to start a differential rqutine wherein only extremals are used. A procedure is given for this, together with a measure of the variation of any curve from an extremal. The measure has no obvious significance except that it is zero whenever the curve is an extremal and is positive otherwise.

\section{Basic Equations}

the form

The equations which govern the system are assumed to have

$$
\dot{\mathrm{x}}^{1}=\mathrm{f}^{1}, \quad 1=1, \cdots, \mathrm{n},
$$

where $f^{i}=f^{i}(x, p, t), \quad x=\left(x^{i}\right)$ is an $n$-dimensional vector $\mathrm{p}=\left(\mathrm{p}^{\sigma}\right)$ is an m-dimensional vector, and $t$ is a scalar which we may think of as time. The set $\left(x^{i}\right)$ are called state variables and the set $\left(p^{\sigma}\right)$ are called control variables. The $p^{\prime}$ 's are to be chosen as functions of time to effect whatever optimum is desired. Vectors may be written in index notation $x^{1}$, in vector notation $\bar{x}$ or in matrix notation ( $x$ ) or $\left(x^{i}\right)$, whichever suggest the important properties. Partial derivatives will be denoted by subscripts when no error seems likely; for example $f_{j}^{i} \partial f^{i} / \partial x^{j}$ and $f_{\sigma}^{i}=\partial f^{i} / \partial p^{\sigma}$; Latin indices will have the ${ }^{j}$ range $1-n$ and Greek indices will have the range $1-\mathrm{m}$. It will be assumed that whatever derivatives occur are continuous.

Some familiarity with calculus of variations is assumed. A typical problem is that of going from one specified point to another, from $\left(x_{0}\right)$ to $(X)$ or $\left(X_{T}\right)$ in such a way that the terminal value $T$ of the time is a minimum. The form of the equations involved is the same, independent of the particular problem. The variational equations are

$$
\delta \dot{x}^{i}=f_{j}^{i} \delta x^{j}+f_{\sigma}^{i} \delta{ }_{p}^{\sigma}
$$

(the summation signs will be omitted if no error seems likely), and the associated adjoint system of equations for the Lagrange multipliers is

$$
\dot{\lambda}=-\mathbf{f} \lambda_{j} \text {. }
$$

The function

$$
H=\lambda_{1} f^{i}=\bar{\lambda} \cdot \bar{f}
$$

is called the hamiltonian. The Euler equations

$$
H_{\sigma}=0=\lambda_{1} f_{\sigma}^{1}
$$

are necessary for an optimum if $p$ lies in an open region. An admissible path is one which begins and ends at the 

specified initial and terminal points. The fundamental lemma states that if we have an admissible path on which Eq. (1.5) is not satisfied, then we can construct a better admissible path. The principal difficulty is in matching end conditions, in solving a two-, or multi-point boundary problem.

We shal1 need two fundamental sets for the adjoint system. In the first one, the initial conditions are given: $\bar{\lambda}_{j}$ or $\left(\lambda_{1 j}\right)$ are chosen so that $\lambda_{i j}(0)=\delta_{i j}$ (the Kronecker delta). The other set $\bar{\lambda}^{j}$ is chosen so that at the terminal time $T$, or an approximation to $i t, \lambda j_{i}^{j}=\delta_{i}^{j}$. We need those with initial values to start integration, but the formulas are neater when the $\pi^{j}$ are used. For any particular path they are functions of one another, as follows. Let $L$ be the matrix with elements $L_{i j} \neq \lambda_{j i}(T)$. It is not singular. Let $L^{-1}$ be $M$, with elements $M^{i j}$. Then $\bar{\lambda}^{i}=M^{i j} \bar{\lambda}_{j}$, as we see from

$$
\lambda_{j}^{i}(T)=\delta_{j}^{i}=M^{i k} \lambda_{j k}(T)=M^{i k} L_{k j} \text { : }
$$

There is also the Green:'s formula for the variations of the end values of the state variables, as given by Bliss ( 8 ),

$$
\delta x^{1}(T)=\int_{0}^{T} \bar{\lambda}^{i} \cdot \bar{f}_{\sigma} \delta_{p}{ }^{\sigma} d t
$$

as a consequence of Eqs. (1.2) and (1.3). The corresponding differentials are

$$
d x^{i}(T)=\delta x^{i}(T)+\dot{x}^{1}(T) d T .
$$

let

Finally, let us consider a special set of variations;

Then

$$
\delta_{\mathrm{p}}^{\sigma}+\Sigma c_{1} \bar{\lambda}^{1} \cdot \overline{\mathbf{f}}_{\sigma} \text {. }
$$

$$
\delta x^{i}(T)=\int_{0}^{T} c_{j} \bar{\lambda}^{j} \cdot \bar{f}_{\sigma} \bar{\lambda} \bar{t}_{\sigma} d t
$$

which has the form

$$
\delta x^{i}=A^{i j} c_{j} ;
$$

The matrix $A$ with elements $A^{i j}$,

$$
A^{i j}=\int_{0}^{\frac{T}{\lambda^{i}} \cdot \bar{f}_{\sigma}} \dot{\bar{\lambda}}^{j} \cdot \bar{f}_{\sigma} d t
$$

is a positive semi-definite (at least) symmetric matrix, since it is a gramian or a sum of gramian matrices.

Since we will generally integrate forward it will be convenient to have this in terms of $\bar{\lambda}^{j}$. If we define the matrix $\propto$ with elements 



$$
A_{i j}=\int_{0}^{T} \bar{\lambda}_{i} \cdot \bar{f}_{\sigma} \bar{\lambda}_{j} \cdot \bar{f}_{\sigma} d t,
$$

then, since $\bar{\lambda}_{i}=L_{i j} \bar{\lambda}^{j}$,

$$
\left.A_{i j}=A^{k} L_{k i} L_{1 j} \quad \text { (or } \alpha=L^{*} A L\right) \text {. }
$$

We shall be concerned almost entirely with problems wherein $A$ and $\alpha$ have rank $n$ except when the path is an extremal, and on extremals they have rank n-1. An extremal is a curve whereon Eq. (1.5) is satisfied. Implicit in the above relations is that there are no corners.

\section{Method of the Fundamental Lemma}

In this section a variant of the $B F$ method is given which involves only forward integration. The essential of this method is that there are two sets of corrections or variations generated. The first set drives the path toward admissibility and the second is orthogonal to these and drives the path toward an extremal.

Let us consider the minimum time problem as a typical problem. The initial point $\left(x_{0}^{i}\right)$ and the terminal point $\left(x^{i}\right)$ are specified and we wish to minimize the terminal value $T$ of $t$. Let us choose a likely path by choosing or generating in some fashion the control variable. Let us compute the trajectory, storing the control variable and a fundamental set of solutions for the adjoint system. Let us calculate also the integrals $A_{i j} \cdot$ We can invert the matrix $L$ and get $A^{i j}$. If we consider the special variations of Eq. (1.9), then by Eq́s. (1.7), (1.8), and (1.12) we get

$$
d x^{i}(T)=A^{i j} c_{j}+\dot{x}^{i}(T) d T \text {. }
$$

If we treat differentials as differences, we get

$$
x^{i}-x^{i}(T)=A^{i j} c_{j}+\dot{x}^{i}(T) \Delta T \text {. }
$$

This is a set of $n$ equatons for $n+1$ unknowns $c_{1}, \cdots$, $c_{n}, \Delta T$.

Let us now choose a set of c's to drive the path toward admissibility. Let us examine the $n$ matrices obtained from the $n^{\text {:by }} \mathrm{n}+1$ matrix $\left(\mathrm{A}^{i j}, \dot{x}^{i}\right)$ of Eq. (2.2) by omitting the first $n$ columns one at a time. Let us choose the one of these which has the largest determinant. Assume for definiteness that we omit the n'th column. Let us then solve

$$
\begin{aligned}
x^{i}-x^{i}(T)=A^{11} c_{11}+A^{i 2} c_{21} & +\cdots+A^{1 n-1} c_{n-11} \\
& +\dot{x}^{i} \Delta T .
\end{aligned}
$$



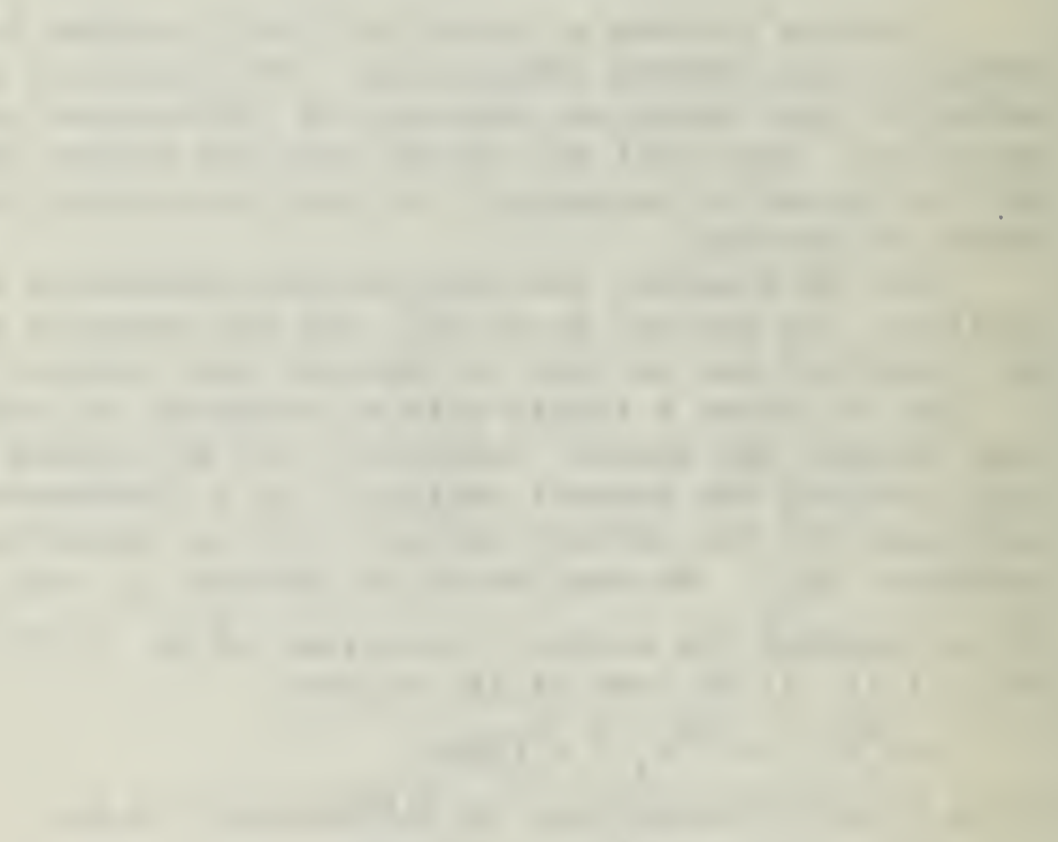

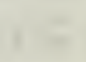


If we choose $c_{n 1} \leqslant 0$ then this set of variations tends to make the resulting path admissible. Now let us choose a second set of variations with $c_{n 2} \leftarrow \epsilon$, satisfying the equations

$$
0=A^{i j} c_{j 2}+\dot{x}^{i} \Delta T_{2} \text {. }
$$

The first-order effects of this do not change the admissibility of the path. The set $c_{12}, c_{22}, \cdots, c_{n-1}, \Delta T_{2}$ are linear in $\epsilon$ : If $\Delta \mathrm{T}_{2} / \epsilon$ is positive, choose $\epsilon$ negative and vice versa. A minor problem is to choose $\epsilon$ well. If it is chosen too large, the corrections overshoot and if it is too small, the sequence creeps toward the desired extremal.

This routine has the advantage that it avoids the alternate forward and backward integration of the original method. This is at the expense of computer storage. It also avoids a term that to the author was puzzling, the quantity in the radical for choosing the step size. The indeterminancy in choosing the step size is inherent in the method unless the operator has some feel for the problem.

Storage of the adjoint variables, for correcting the control variables máy be eliminated by using the values of the c's found in one iteration with the solutions to the adjoint generated in the next.

\section{Differential Method}

In this method only extremals are used. The Euler equation, Eq. (1.5) will be assumed to be satisfied identically on every path considered. Implicitly then, the control variable maximizes $H$ at an interior point. The problem is that the maximization occurs for some unknown solution

$$
\bar{\lambda}=c^{i} \bar{\lambda}_{i}
$$

to the adjoint. Now suppose that we have been able to get an estimate of the C's for the desired extremal. It happens that we may choose one $C$, say $C_{n}$, arbitrarily, since the essential equations are homogeneous in the $\bar{\lambda}^{\prime} s$; we must only be certain that the choice has the right sign.

Now let:us consider the effects of changing the C's. Let us change the C's and the control variables, assuming that we can do this leaving $x, t$ fixed. From Eqs. (1.5) and (3.1) we get

$$
\bar{\lambda}_{i} \cdot \bar{f}_{\sigma} \mathrm{dc}^{i}+\bar{\lambda} \cdot \overline{\mathrm{f}}_{\sigma T} \delta \mathrm{p}^{\top}=0
$$

a term involving $\delta x$ has been omitted from these equations, under the assumption that $x, t$ could be held fixed. We can solve this equation for $\delta_{p}$ in terms of $d C$ provided the $m^{\prime} t h$ 


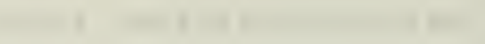
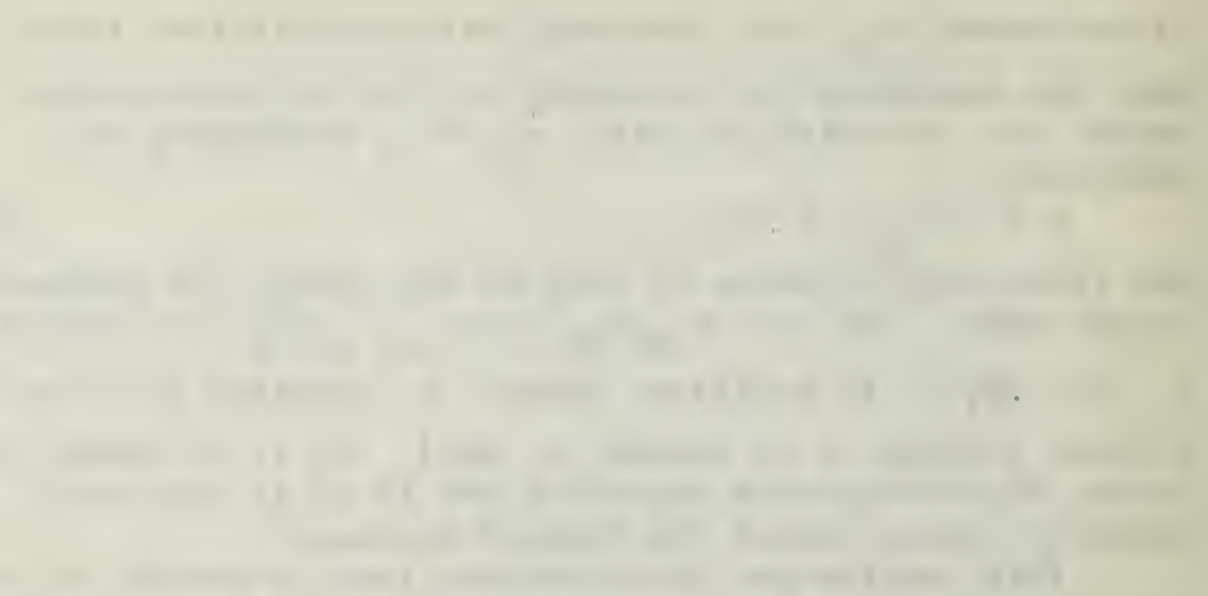

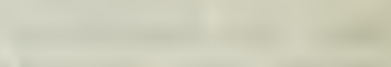
.
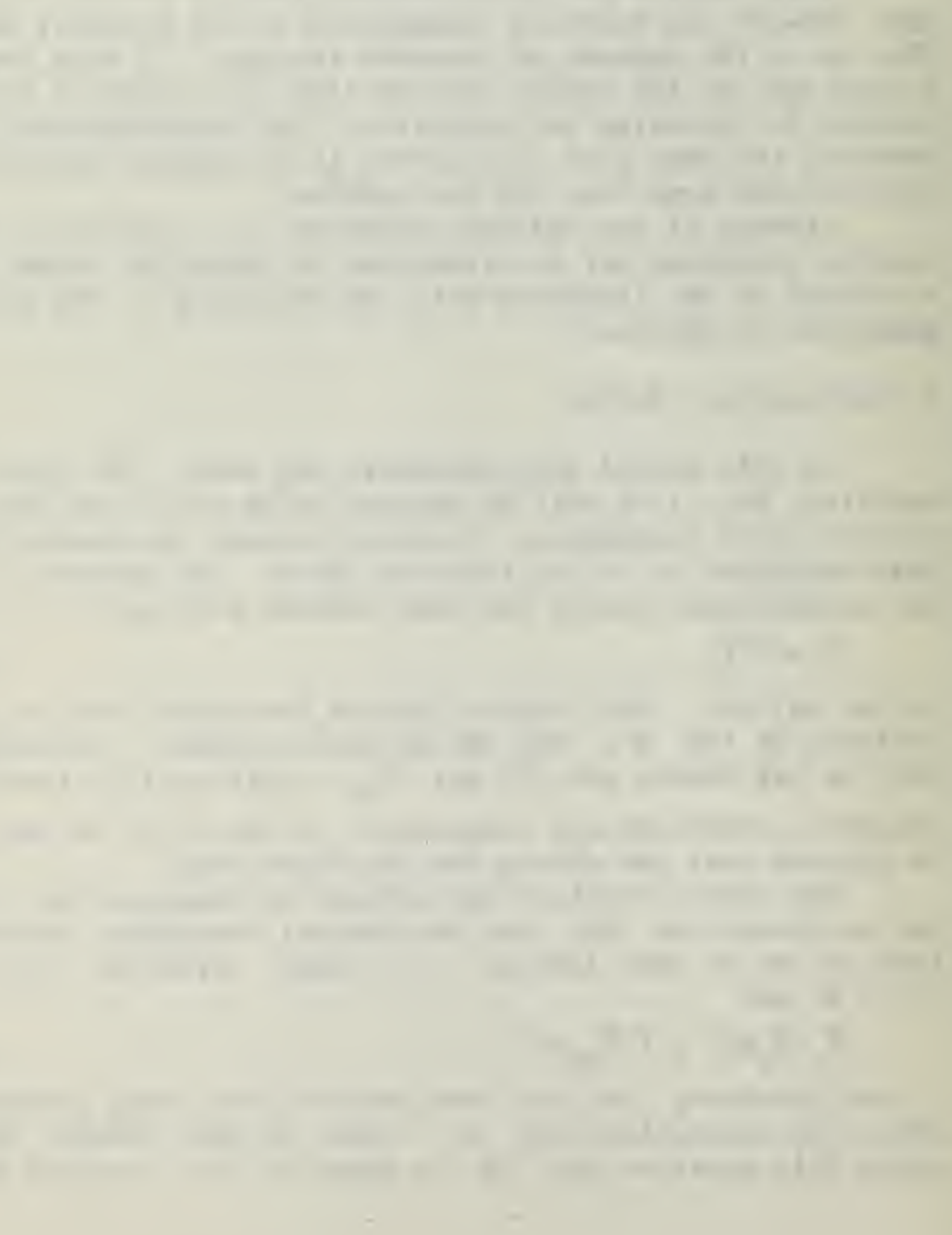
order determinant with elements $\bar{\lambda} \cdot \bar{f}_{\sigma \tau}$ is not zero, and substitute into Eqs. (1.7) and (1.8) to get $n$ equations of the form

$$
\mathrm{dx}^{1}(\mathrm{~T})=\sum_{1}^{\mathrm{n}-1} \mathrm{~B}_{\mathrm{j}}^{\mathrm{i}} \mathrm{dC}^{j}+\dot{\mathrm{x}}^{\mathrm{i}}(\mathrm{T}) \mathrm{dT} ;
$$

the $B^{i}$ are integrals. This is the basic relation for setting up the Newton-Raphson iteration. We run a trajectory, calculating the integrals $B \frac{i}{j}$; we treat differentials as differences; we substitute $\mathrm{X}^{i}-\mathrm{x}^{i}(\mathrm{~T})$ for $d \mathrm{x}^{i}$ and solve for $\Delta \mathrm{C}^{i}$, $\Delta \mathrm{T}$.

The principal problem is that of convergence. Various problems involving discontinuous control have been solved by this method. A term must be added in the differential formulas for each corner where the value of $t$ is not specified, and corner conditions must be met. It seems to the author that if the path has corners the programmer must anticipate this, since the control variable is discontinuous. Indeed the difficulties in finding the roots of algebraic equations suggest that no method exists for checking the Weierstrass condition genera $11 y$.

\section{Determining a 'Best-fitting' Extrema1.}

The studies have indicated that it is easier to develope a fairly good path with FL methods, but it is not clear how nearly an extremal has been approached. The differential method uses only extremals and tends to converge exponentially when it converges, but it is harder to get a satisfactory approximation for starting. This suggests that we might use an FL method initial1y, and then use it to determine a 'bestfitting' extremal, to continue with the differential method.

The problem then becomes: how can we get a best-fitting extrema 1 for the path just found? The answer lies in the method of correction for the FL method. Let us consider the matrix A defined by Eq. (1.12). As a gramian or the sum of gramiansit is symmetric and positive definite, or at least semi-definite. Hence its eigenvalues are either positive or zero and its eigenvectors are orthogonal, or in case of equal eigenvalues can be chosen so. write it

Now let us consider Eq. (2.1) for fixed $T$. We may

$$
(\delta \mathrm{x})_{\mathrm{T}}=\mathrm{A}(\mathrm{c})
$$

Generally $A$ has rank $n$ but for extremals it has rank $n-1$. Let us assume this to be the case in the following.

Then, by Eq: (4.1) we can attain at time $T$ any point in some neighborhood of the endpoint of a curve which is not an extremal, but the endpoint of extremals define an $n-1$ disc 

in n-space. We have seen that with an extremal is a solution to the adjoint $\bar{\lambda}$, say $\lambda *$. If $E *$ is the extremal, embedded in a family (E) of extremals starting from ( $\left.x_{0}^{1}\right)$, then for fixed $T, \bar{\lambda} *(T)$ is normal to the disc defined by the endpoints of (E).

Now consider the corrective routine and the matrix $A$. As the successive iterates approach an extremal A becomes singular. Let $0 \leqq \gamma_{1}<\gamma_{2} \leqq \cdots \leqq \gamma_{n}$ be the eigenvalues of $A$, and assume that $\gamma_{1} \ll \gamma_{2}$; Let $\bar{\lambda}_{\mathrm{A1}}, \cdots, \bar{\lambda}_{\mathrm{An}}$ be the corresponding eigenvectors of $A$. It is clear that if we had attained the extremal, so that A were singular, then

$$
\bar{\lambda} *(\mathrm{~T}) \| \bar{\lambda}_{\mathrm{Al}} \text {. }
$$

But $\bar{\lambda} *$ is only determined to within a multiplicative factor. Hence $\bar{\lambda} *(T)$ is determined as the eigenvector corresponding to the zero eigenvalue of $A$. We may get $\bar{\lambda} *(0)$ from the matrix $L$ defined in the first section. Most computing centers have routines for finding the eigenvalues and eigenvectors of a symmetric matrix.

This suggest a measure of approximation to an extremal, either $\gamma_{1} / \gamma_{2}$ or $\gamma_{1} / \gamma_{n}$, the ratio: of the smallest eigenvalue to either the next smallest or to the largest. Unfortunately it seems to have just one important property; it becomes zero when an extremal is attained.

The same procedure works equally well on $\alpha$. When the curve approaches an extremal, the eigenvector corresponding to the zero eigenvalue gives the starting values for the adjoint. If the path is not an extremal, there seems to be no simple relation among the eigenvalues and eigenvectors of $A$ and $\alpha$, since the only property of the matrix $L$ is that its determinant is positive.

\section{Comments}

Considerable computation has been done in an attempt to compare the two methods, without a definite conclusion. Seibel (9) made comparisons mainly in the ship-routing problem wherein convergence is not a serious problem. Usually the time required for determining a course by the FD method was about two-fifths or half that for the BFL method:

Considerable work was done intrying to compare the two methods (10) on the problem of maximum range for a high-speed glider. The problem is on the verge of instability in two ways. First, a slight increase in initial velocity puts it virtually in orbit. Second, the range is apparently extremely sensitive to the value used for the radius of the earth. An 


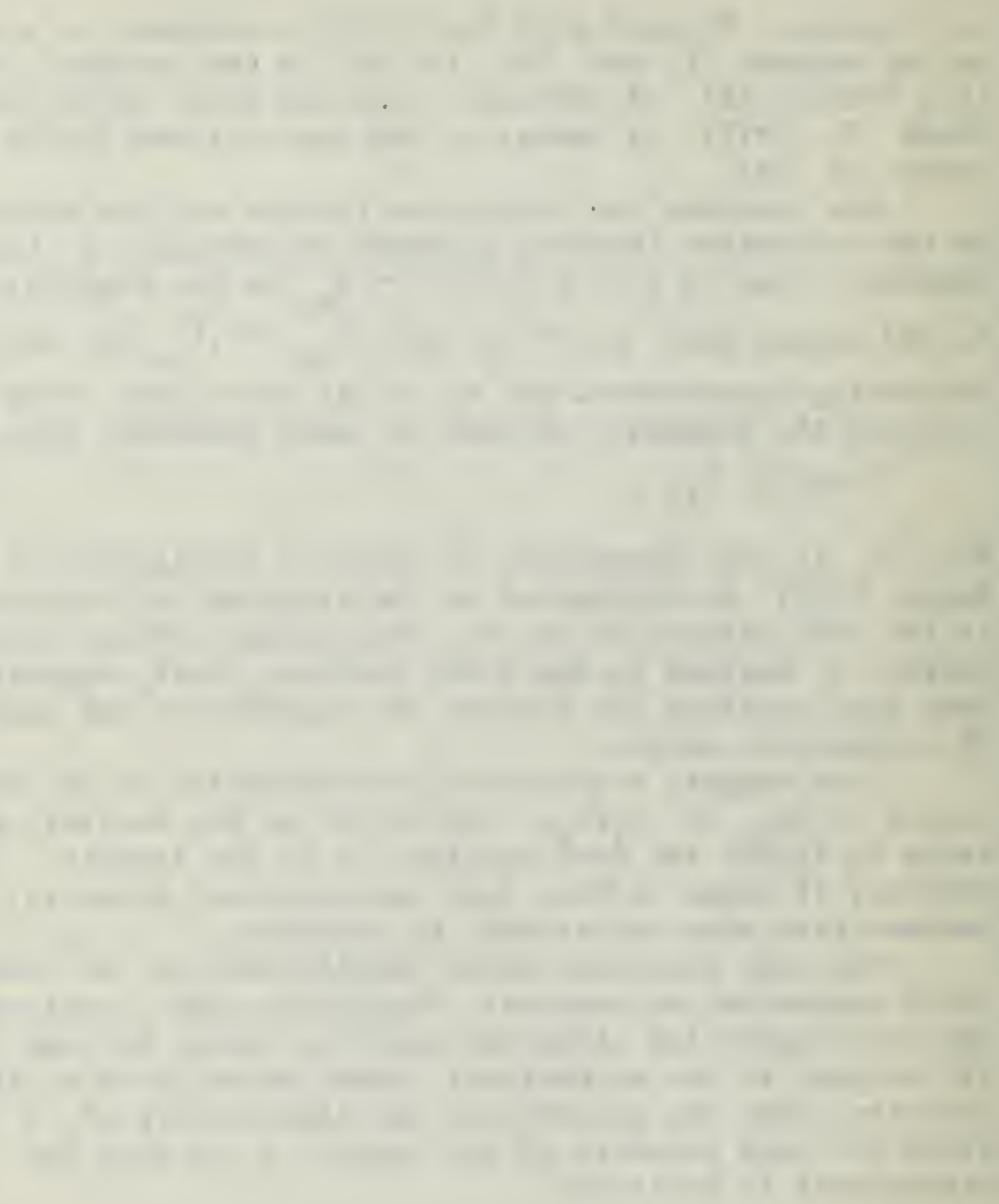


increase of about thirty NM (nautical miles) in the value used caused an increase in range from about 39,000 NM to about 54,000 NM. These are reflected in very large values for the Lagrange multipliers, one of them being about $10^{12}$ times as large as another initially. Discussions suggest that this might be overcome by the adoption of a double-precision routine like; that used by Johnson (11) in a related problem. The latest computations actually carried out gave a much better trajectory by the BFL method in the last moments of the trajectory. It does not seem possible, even knowing the initial values of the Lagrange multipliers very closely, to compute the complete trajectory for the desired extremal; it always seems to end in a loop. The ratio of the Lagrange multipliers which are so iarge initially determines the angle of attack. In the last moment these must both be driven to zero. This could not be done. It might be pointed out that this problem is very stable in another sense. It seems possible to get good open-loop programs and very good closed-loop programs, these leading to ranges quite near the maximum. Different models were used for the atmosphere, but a few computations suggested that this was not a significant factor.

The FD method differs from the so-called method of second variations given by Breakwe11, Speyer, and Bryson (12) in that the variations of the state variables were dropped in Eq. (3.2). It seems to the author that the name secondvariation given to that method is a misnomer. The coefficients in the linear forms of Eq. (3.2) are the same as the coefficients in the Legendre quadratic form but they are not second variations.

The important thing is that we can calculate differentials associated with extremals. This method has been applied to several problems by the author and his students where no terms appeared corresponding to the second derivatives. McCalla (3) in his thesis treated a low-order bang-bang problem. Faulkner and Ward (5) treat the problem of ballistic missile interception where bounded control and limited energy expenditure were imposed. Professor Bleick and the author treat a launching problem where there is an intermediate period of coasting. In these there are corners at points which are unknown initially; no way is seen to extend the method of the fundamental lemma to these, since the proof assumes that 'two-sided' corrections to the control are a1lowed. Others have advocated the use of 'slack' variables in posing problems such as these. It is the author's strong opinion that if the problem can be posed without these, its solution 18 simpler without them.

The author felt perhaps that some fo the convergence problems were due to the omission of the terms. This is not always the case, since the two methods coincide 

when the equations are linear in the state variables and serious convergence problems were encountered in the problem of rendezvous in minimum time, with linearized equations

The FD method also applies to the solution of some allocation problems of the following type. An integral of the form

$$
G=\int_{0}^{T} f(t, p, q) d t
$$

is to be maximized with respect to $p$ and minimized with respect to $q$, which are probability density functions (13). It is easily posed as a simple linear control problem involving a third order system with limited total input, being unusual in that the solution is of minimax type.

\section{References}

(1) Arthur E. Bryson and Walter F. Denham, A steepest-ascent method for solving optimum programming problems, J. Applied Mechanics, p. 247f, June 1962.

(2) Frank D. Faulkner, Determining optimum ship routes, JORSA, 10 , No. 6, 799-807, Nov-Dec 1962.

(3) Thomas R. McCalla, Application of the adjoint system of differential equations in the solution of the bang-bang control problem, Thesis, U. S. Naval Postgraduate School, 1961.

(4) Frank D. Faulkner, Direct methods, Ch. 2 in Optimization Techniques, George Leitmann,Editor, Academic Press, New York, 1962 .

(5) Frank. D. Faulkner, Optimum interception of a bal1istic missile at intermediate range, U. S. Naval Postgraduate School . Research Paper No. 29, October, 1961.

(6) W. E. Bleick and F. D. Faulkner, Orbital transfer with minimum fue1, U. S. Naval Postgraduate School Research Paper No. 40, Sept 1963.

(7) R. L. Kelley, Method of gradients, Ch. 6 in Direct Methods, Beorge Leitmann, Editor, Academic Press, New York, 1962

(8) G. A. Bliss, Mathematics for Exterior Ballistics, Chicago, 1944, Chapter IV.

(9) W. E. Seibe1, A comparison of two methods for solution of the problem of Bolza, Thesis, U. S. Naval Postgraduate School, 1963.

(10) W. W. McCue and R. C. Good, A comparison between steepestascent and differential correction optimization methods in a problem of Bolza, with a method for obtaining starting values for the adjoint variables from a nominal path, Thesis, U. S. Nava1 Postgraduate Schoo1, 1963.

(11) R. G. Johnson, Linear perturbational control for re-entry, presented at joint AIAA-IMS-SIAM-ONR Contro1 Conference, 

Monterey, January 1964.

(12) J. V. Breakwe11, J. L. Speyer, and A. E. Bryson, Optimization and control of nonlinear systems using the second varia: tion, SIAM J. of Contro1, No 2, 1963.

(13) Robert 'H. Bartley, A method for obtaining the solution to some allocation problems, Thesis, U. S. Naval Postgraduate School, (in preparation). 





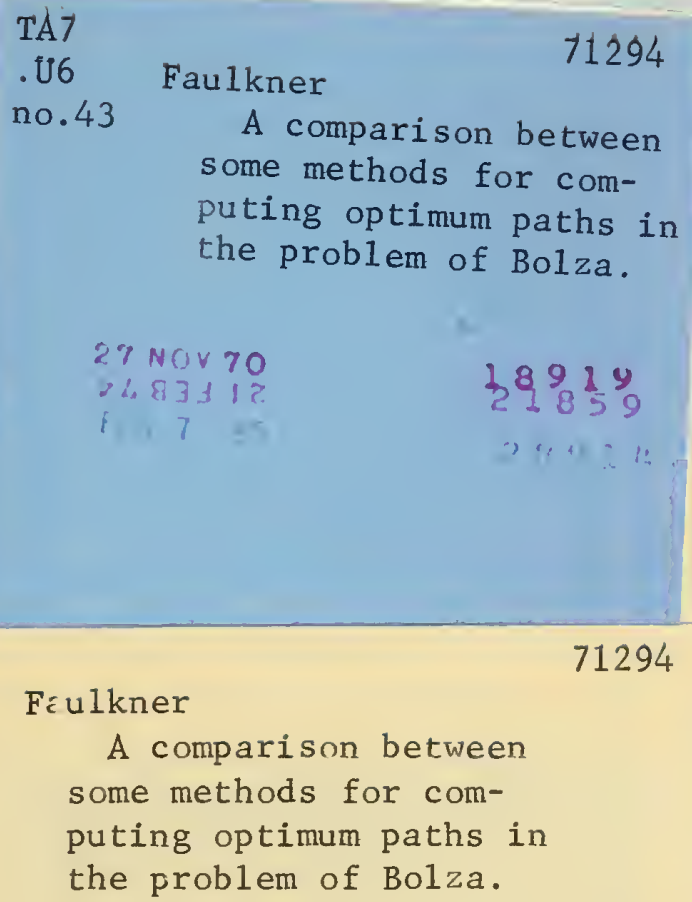


genTA 7.46 no. 43 Los Angeles
A comparison between

A comparison between some methods for co

I.I.

32768002166167

DUDLEY KNOX LIBRARY 